\title{
On the numerical solution of nonlinear integral equation arising in conductor like screening model for realistic solvents
}

\author{
Maryam Attary ${ }^{1}$
}

Received: 6 May 2018 / Accepted: 24 July 2018/Published online: 7 August 2018

(C) The Author(s) 2018

\begin{abstract}
The aim of this work is to introduce an efficient algorithm for the numerical solution of nonlinear integral equation arising from chemical phenomenon which is a famous equation in chemistry engineering. A procedure is described for transforming the nonlinear integral equation by using Chebyshev polynomials to nonlinear system of algebraic equations. Also, we present a convergence analysis and error bound for presented method. In addition, some numerical results are reported to evaluate the validity and applicability of the method and also comparison has been done with existing results.
\end{abstract}

Keywords Hammerestein integral equation - Chemical phenomenon - Chebyshev polynomials · Numerical treatment

\section{Introduction}

Nonlinear form of integral equations of the second kind often occur as following

$u(t)=f(t)+\int_{a}^{b} k(t, s) G(s, u(s)) \mathrm{d} s$,

where $f, k$ and $G$ are known functions with $G(s, u)$ nonlinear in $u$, and $u(t)$ is a solution to be determined. These types of equations can be obtained by reformulation of some boundary value problems for ordinary differential equations, also mixed type of these equations can be seen in chemical reactor as the dynamic model (see e.g., $[2,3,7,8,10,12-14])$. In this article, we study the numerical solution for a special kind of (1.1)

$u(t)=f(t)+\int_{a}^{b} k(t, s)(u(s))^{-1} \mathrm{~d} s$.

Above equation can be written as the following integral equation forms

Maryam Attary

maryam.attari@kiau.ac.ir

1 Department of Mathematics, Karaj Branch, Islamic Azad University, Karaj, Iran $u(\delta)=\int_{a}^{b} k\left(\delta, \delta_{1}\right)\left(u\left(\delta_{1}\right)\right)^{-1} \mathrm{~d} \delta_{1}$

where

$$
u(\delta)=\exp \left(\frac{-\mu_{s}(\delta)}{R T}\right), \quad k\left(\delta, \delta_{1}\right)=P_{s}\left(\delta_{1}\right) \exp \left(\frac{-E_{\mathrm{int}}\left(\delta, \delta_{1}\right)}{R T}\right) .
$$

Substituting (1.4) in (1.3) and through simple computations, we get the following integral equation which appeared in chemical phenomenon

$$
\mu_{s}(\delta)=-R T \ln \left[\int P_{s}\left(\delta_{1}\right) \exp \left(-\frac{E_{\mathrm{int}}\left(\delta, \delta_{1}\right)-\mu_{s}\left(\delta_{1}\right)}{R T}\right) \mathrm{d} \delta_{1}\right],
$$

the last equation forms the basis for the Conductor like Screening Model for Realistic Solvents (COSMO-RS) where $R$ and $T$ are the gas constant and the temperature, respectively, the interaction energy expression for the segments with screening charge density $\delta$ and $\delta_{1}$ has been explained by $E_{\text {int }}\left(\delta, \delta_{1}\right), P_{s}(\delta)$ is the molecular interaction in solvent and $\mu_{s}(\delta)$ denotes the chemical potential of the surface segments that should be determined.

In 1995, A. Klamt achieved an approach that made it possible to compute the details of molecules quantum mechanically and he applied obtained results in an approximate statistical mechanics procedure which is called COSMO-RS. COSMO-RS is a quantum chemistry-based equilibrium thermodynamics method and it can show the 
link between the world of chemical quantum mechanics and engineering thermodynamics well. For the prediction of activity coefficients in multicomponent liquid mixtures, COSMO-RS uses conductor screening charges on molecular surface panels. Detailed description of these models may be found in [17].

The numerical solvability of nonlinear integral equations has been studied by several authors. Ghoreishi et al. [6] have been concerned with the numerical solution of Volterra-Hammerstein integral equations via the operational Tau method. Maleknejad et. al. in [9, 11] applied the computational method for Hammerstein integral equations based on Bernstein operational matrices and operational matrices of hybrid functions. In [12], the Sinc-quadrature formula are applied to approximate solution of (1.4). In [5], numerical solution of nonlinear Hammerstein integral equations via collocation method based on double exponential transformation is considered. Authors of [15] applied operational matrices of Chebyshev polynomials for solving singular Volterra integral equations. Our approach is to investigate how numerically one can solve a COSMORS integral equation with the Chebyshev polynomials.

To clarify the concepts of the Chebyshev polynomials, in "Preliminaries and notations" section we describe some notations and basic definitions of Chebyshev polynomials. We will apply Chebyshev polynomials for approximating the solution of nonlinear integral Eq. (1.2), then convergence analysis and error bound is proved in "Main result" section . Finally in "Numerical" section, some numerical experiments are reported to illustrate the efficiency and accuracy of our algorithm.

\section{Preliminaries and notations}

In this section, we recall some necessary mathematical preliminaries and definitions of the Chebyshev polynomials, which are used further in this paper [1, 4].

Definition 1 The Chebyshev polynomials of the first kind of order $i$ are defined as follows

$$
T_{i}(t)=\cos \left[i \cos ^{-1} t\right], \quad t \in[-1,1], \quad i=0,1,2, \ldots,
$$

from this definition the following property is evident

$$
T_{i}(\cos \theta)=\cos (i \theta), \quad \theta \in[0, \pi], \quad i=0,1,2, \ldots
$$

Theorem 1 The Chebyshev polynomials $T_{i}(t)$ satisfy the recurrence relation

$T_{i+1}(t)=2 t T_{i}(t)-T_{i-1}(t), \quad i=1,2, \ldots$

Proposition 1 Chebyshev polynomials are orthogonal functions with respect to a weighting function of $\left(1-t^{2}\right)^{-1 / 2}$, $\int_{-1}^{1} T_{i}(t) T_{j}(t)\left(1-t^{2}\right)^{-1 / 2} \mathrm{~d} t=\frac{\pi}{2} c_{i} \delta_{i j}$,

where

$c_{i}=\left\{\begin{array}{ll}2 & i=0, \\ 1 & i>0,\end{array} \quad\right.$ and $\quad \delta_{i j}=\left\{\begin{array}{cc}0 & i \neq j, \\ 1 & i=j .\end{array}\right.$

\section{Main result}

In this section for the sake of clarity, we prefer to split our presentation into two parts. The first part concerns with numerical scheme of nonlinear integral Eq. (1.2) based on the Chebyshev polynomials. The second part deals with convergence analysis of the presented method.

\section{Nonlinear function approximation and Chebyshev collocation method}

Using defined bases, orthogonal series expansion of the exact solution $u(t)$ can be considered as

$u(t)=\sum_{i=0}^{\infty} a_{i} \varphi_{i}(t)$

where $a_{i}=<u(t), \varphi_{i}(t)>$.

Let $\varphi(t)$ be a given set of orthogonal bases with respect to the defined weight function $\omega(t)$ on $[a, b]$. Owing to variety of orthogonal polynomials, in the following we choose Chebyshev polynomials. Now, we define an approximation function of the exact solution $u(t)$ as follows

$u(t) \simeq \sum_{i=0}^{n} a_{1 i} T_{i}(t)=\mathbf{A}_{1}^{\mathrm{T}} \mathbf{T}_{i}(t)$

where $\quad \mathbf{A}_{1}=\left[a_{1,0}, a_{1,1}, \ldots, a_{1, n}\right]^{\mathrm{T}}, \quad$ and $\quad \mathbf{T}_{i}(t)=\left[T_{0}(t)\right.$, $\left.T_{1}(t), \ldots, T_{n}(t)\right]^{\mathrm{T}}$.

Without loss of generality and due to COSMO-RS Eq. (1.5), the nonlinear analytic function in (1.1) can be written as

$F(t)=G(t, u(t))=(u(t))^{-1}$,

according to (3.2), we can write (3.3) as

$F(t)=\mathbf{A}_{2}^{\mathrm{T}} \mathbf{T}_{i}(t)$,

where $\mathbf{A}_{2}=\left[a_{2,0}, a_{2,1}, \ldots, a_{2, n}\right]^{\mathrm{T}}$.

By substituting Eqs. (3.2)-(3.4) in (1.1), or (1.2), we have

$\mathbf{A}_{1}^{\mathrm{T}} \mathbf{T}_{i}(t)=f(t)+\int_{a}^{b} k(t, s) \mathbf{A}_{2}^{\mathrm{T}} \mathbf{T}_{i}(s) \mathrm{d} s$.

Let us set $\mathbf{H}(t)=\int_{a}^{b} k(t, s) \mathbf{T}_{i}(s) \mathrm{d} s$. Using this notation, above equation can be transformed to the following form 
$\mathbf{A}_{1}^{\mathrm{T}} \mathbf{T}_{i}(t)=f(t)+\mathbf{A}_{2}^{\mathrm{T}} \mathbf{H}(t)$.

On the other hand, due to (3.2)-(3.4) we can write

$\mathbf{A}_{2}^{\mathrm{T}} \mathbf{T}_{i}(t)=G(t, u(t))=G\left(t, \mathbf{A}_{1}^{\mathrm{T}} \mathbf{T}_{i}(t)\right)$,

from (3.6), above relation can be rewritten as

$$
\mathbf{A}_{2}^{\mathrm{T}} \mathbf{T}_{i}(t)=G\left(t, f(t)+\mathbf{A}_{2}^{\mathrm{T}} \mathbf{H}(t)\right),
$$

for obtaining $(n+1)$ unknowns $\mathbf{A}_{2}$, we take $t=t_{j}$ for $j=1,2, \ldots,(n+1)$, where $t_{j}$ be collocation points. So, we have the following nonlinear system

$\mathbf{A}_{2}^{\mathrm{T}} \mathbf{T}_{i}\left(t_{j}\right)=G\left(t_{j}, f\left(t_{j}\right)+\mathbf{A}_{2}^{\mathrm{T}} \mathbf{H}\left(t_{j}\right)\right)$.

By solving Eq. (3.9), $\mathbf{A}_{2}$ can be determined. Finally, the approximation solution $u(t)$ will be obtained

$u(t)=\frac{1}{F(t)}=\frac{1}{\mathbf{A}_{2}^{\mathrm{T}} \mathbf{T}_{i}(t)}$.

The following algorithm summarizes our proposed method: approximation function of the exact solution $g(t)=\sum_{i=0}^{\infty} a_{i} T_{i}(t)$. Then, for sufficiently smooth functions $g(t)$ and $k(t, s)$, with $|k(t, s)| \leq M$, in (1.2) we have

$$
\begin{aligned}
& \left\|u(t)-u^{*}(t)\right\|_{L_{\infty}[-1,1]} \leq 2 M L(b-a) \sum_{i=n+1}^{\infty} \frac{1}{(i-1)^{2}}, \\
& {[a, b] \subseteq[-1,1],}
\end{aligned}
$$

where $u^{*}(t)=\frac{1}{g^{*}(t)}$.

Proof Due to Eq. (1.2) we can write

$$
\begin{aligned}
\left\|u(t)-u^{*}(t)\right\| & =\max _{t \in[-1,1]}\left|\int_{a}^{b} k(t, s)\left[g(s)-g^{*}(s)\right] \mathrm{d} s\right| \\
& =\max _{t \in[-1,1]}\left|\int_{a}^{b} k(t, s)\left[\sum_{i=0}^{\infty} a_{i} T_{i}(s)-\sum_{i=0}^{n} a_{i} T_{i}(s)\right] \mathrm{d} s\right| \\
& =\max _{t \in[-1,1]}\left|\int_{a}^{b} k(t, s)\left[\sum_{i=n+1}^{\infty} a_{i} T_{i}(s)\right] \mathrm{d} s\right| \\
& \leq \max _{t \in[-1,1]} \int_{a}^{b}|k(t, s)|\left|\sum_{i=n+1}^{\infty} a_{i} T_{i}(s)\right| \mathrm{d} s \\
& \leq \max _{t \in[-1,1]} \int_{a}^{b}\left|k(t, s) \| \sum_{i=n+1}^{\infty} a_{i}\right|\left|T_{i}(s)\right| \mathrm{d} s
\end{aligned}
$$

Algorithm 1. The construction of proposed method for a nonlinear integral equation (1.2)

Step 1. Input:

$$
n, t_{j},(j=1, \ldots, n+1), f(t) \text { and } \mathbf{T}_{i}(t) .
$$

Step 2. Compute $f(t)$ in collocation point $t_{j}$.

Step 3. Compute $\mathbf{H}(t)$ in collocation point $t_{j}$ as follows

$$
\mathbf{H}(t)=\int_{a}^{b} k(t, s) \mathbf{T}_{i}(s) d s .
$$

Step 4. Compute:

4.1. $\mathbf{A}_{2}$ from the following equation:

$$
\mathbf{A}_{2}^{T} \mathbf{T}_{i}\left(t_{j}\right)=G\left(t_{i}, f\left(t_{j}\right)+\mathbf{A}_{2}^{T} \mathbf{H}\left(t_{j}\right)\right) .
$$

4.2. $u(t)$ from relation $u(t)=\frac{1}{\mathbf{A}_{2}^{T} \mathbf{T}_{i}(t)}$.

\section{Error analysis}

This section is concerned with the error estimation of the Chebyshev expansion for the nonlinear integral Eq. (1.2). The following theorem states and proves the main result of error analysis.

Theorem 2 Let $g(t)=\frac{1}{u(t)}$ be a second-order derivative square-integrable function defined on $[-1,1]$ with $\left|g^{\prime \prime}(t)\right| \leq L$ for some constant L,and let $g^{*}(t)$ be an from properties of Chebyshev polynomials and given assumption, we obtain

$$
\left\|u(t)-u^{*}(t)\right\| \leq M(b-a) \sum_{i=n+1}^{\infty}\left|a_{i}\right| .
$$

On the other hand, from $g(t)=\sum_{i=0}^{\infty} a_{i} T_{i}(t)$ we have $a_{i}=<g(t), T_{i}(t)>=\frac{2}{\pi c_{i}} \int_{-1}^{1} g(t) T_{i}(t) \omega(t) \mathrm{d} t$.

Substituting $\omega(t)=\left(1-t^{2}\right)^{-1 / 2}$ and $t=\cos \theta$ yields 


$$
\begin{aligned}
a_{i} & =\frac{2}{\pi c_{i}} \int_{0}^{\pi} g(\cos \theta) T_{i}(\cos \theta)\left(1-\cos \theta^{2}\right)^{-1 / 2} \sin \theta d \theta \\
& =\frac{2}{\pi c_{i}} \int_{0}^{\pi} g(\cos \theta) \cos i \theta \mathrm{d} \theta .
\end{aligned}
$$

Using integration by part, we conclude

$$
\begin{aligned}
a_{i}= & \frac{2}{\pi c_{i} i} \int_{0}^{\pi} g^{\prime}(\cos \theta) \sin i \theta \sin \theta \mathrm{d} \theta \\
= & \frac{1}{\pi c_{i} i} \int_{0}^{\pi} g^{\prime}(\cos \theta)[\cos (i-1) \theta-\cos (i+1) \theta] \mathrm{d} \theta \\
= & \frac{1}{\pi c_{i} i}\left[\int_{0}^{\pi} g^{\prime}(\cos \theta) \cos (i-1) \theta \mathrm{d} \theta\right. \\
& \left.-\int_{0}^{\pi} g^{\prime}(\cos \theta) \cos (i+1) \theta \mathrm{d} \theta\right] .
\end{aligned}
$$

By repeating the integration, $a_{i}$ can be written as

$$
\begin{aligned}
a_{i}= & \frac{1}{\pi c_{i} i}\left[\frac{1}{i-1} \int_{0}^{\pi} g^{\prime \prime}(\cos \theta) \sin (i-1) \theta \sin \theta \mathrm{d} \theta\right. \\
& \left.-\frac{1}{i+1} \int_{0}^{\pi} g^{\prime \prime}(\cos \theta) \sin (i+1) \theta \sin \theta \mathrm{d} \theta\right],
\end{aligned}
$$

thus for $i>1$, (for $i=0, c_{i}=1$ ), we have

$$
\left|a_{i}\right| \leq \frac{1}{\pi i} \int_{0}^{\pi}\left|g^{\prime \prime}(\cos \theta)\right| \mathrm{d} \theta\left(\frac{1}{i-1}+\frac{1}{i+1}\right),
$$

according to assumption and by simple computation, we can write:
Example 1 Consider the following COSMO-RS integral equation which expresses a particular case of the electrostatic misfit energy [12]

$$
u(\delta)=\int_{-3}^{3} P_{s}\left(\delta_{1}\right) \Theta\left(\delta, \delta_{1}\right)\left(u\left(\delta_{1}\right)\right)^{-1} \mathrm{~d} \delta_{1},
$$

where

$$
\begin{aligned}
& P_{s}(\delta)= \\
& \begin{cases}\exp \left(-(5 \delta+0.25)^{2}\right)+\frac{1}{25 \delta^{2}+1}+\frac{\sin (5 \delta+0.25)^{2}}{(5 \delta-0.25)^{4}+1}+q(5 \delta), & -2 \leq \delta<2, \\
0, & \text { Otherwise, }\end{cases}
\end{aligned}
$$

and

$$
q(\delta)= \begin{cases}-(\delta-7)(\delta-9), & 7 \leq \delta<9 \\ 0, & \text { Otherwise }\end{cases}
$$$$
\Theta\left(\delta, \delta_{1}\right)=\exp \left(-\left(\delta+\delta_{1}\right)^{2}\right) .
$$

Here, we apply presented method based on Algorithm 1. This problem has been defined over the interval $[-3,3]$. In order to use Chebyshev polynomials $T_{i}(t)$ on the interval $t \in[a, b]$, we convert it by introducing the change of variable $t=\frac{b-a}{2} x+\frac{b+a}{2}$. For computational details, we take $n=8$ and obtain collocation points $t_{i}$. In this problem $f(t)=0$, so due to step 3 in mentioned algorithm, we compute $H\left(t_{i}\right)$

\begin{tabular}{lllllllll}
\hline$t_{i}$ & -1.61 & -1.11 & -0.61 & -0.11 & -0.39 & -0.89 & -1.39 & -1.89 \\
\hline$H\left(t_{i}\right)$ & 0.052225 & -0.237845 & -0.201385 & 0.139676 & -0.078774 & 0.129343 & -0.044902 & -0.000194 \\
\hline
\end{tabular}

$\left|a_{i}\right| \leq \frac{2 L}{\left(i^{2}-1\right)} \leq \frac{2 L}{(i-1)^{2}}$.

Finally, by substituting (3.12) in (3.11) we can conclude:

$\left\|u(t)-u^{*}(t)\right\| \leq 2 L M(b-a) \sum_{i=n+1}^{\infty} \frac{1}{(i-1)^{2}}$,

the proof is completed.

\section{Numerical}

In this section, three test problems will be considered to assess the accuracy and efficiency of the proposed method for solving nonlinear integral Eq. (1.2). Achieved nonlinear systems will be solved by Newton method and all calculations will be done by the Mathematics.
From step 4 in Algorithm 1, we solve achieved nonlinear system by Newton method and we obtain $A_{2}$ as

$$
\begin{aligned}
A_{2}= & {[1.36555,-0.17877,0.45421,-0.02481,0.03827,} \\
& -0.00621,0.00471,0.00033]^{\mathrm{T}} .
\end{aligned}
$$

Finally, the approximation solution $u(t)$ will be obtained. Obtained numerical results can be seen in Figs. 1 and 2 with $n=8,16$, respectively. Example 1 was solved in $[9,16]$ by a method based on Sinc, Bernestein polynomials and Block pulse functions. In [16] reported results show that they can achieve good numerical results for $n \geq 40$, whereas reported results using proposed method show that we can achieve good numerical results for $n \geq 8$. Figure 1 of the proposed method shows that high accuracy obtained for $n=8$ in comparison with figure of Sinc method in [9]. 


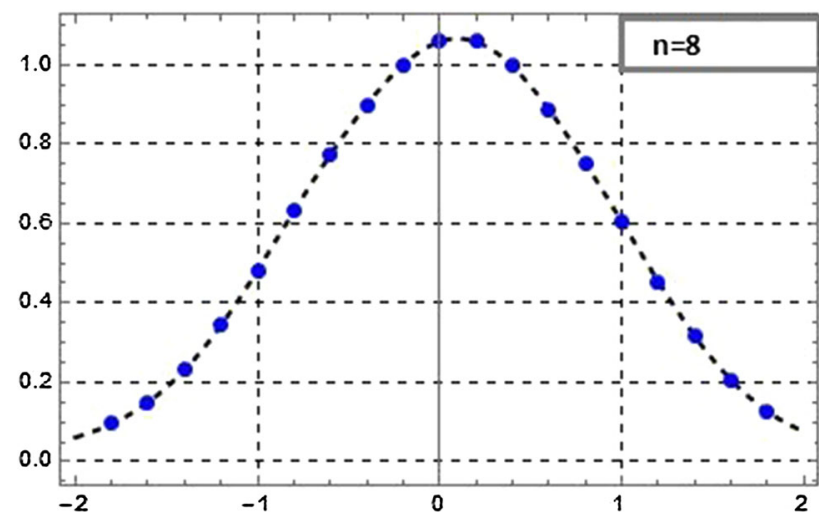

Fig. 1 Approximate solution for Example 1 using Chebyshev base with $n=8$

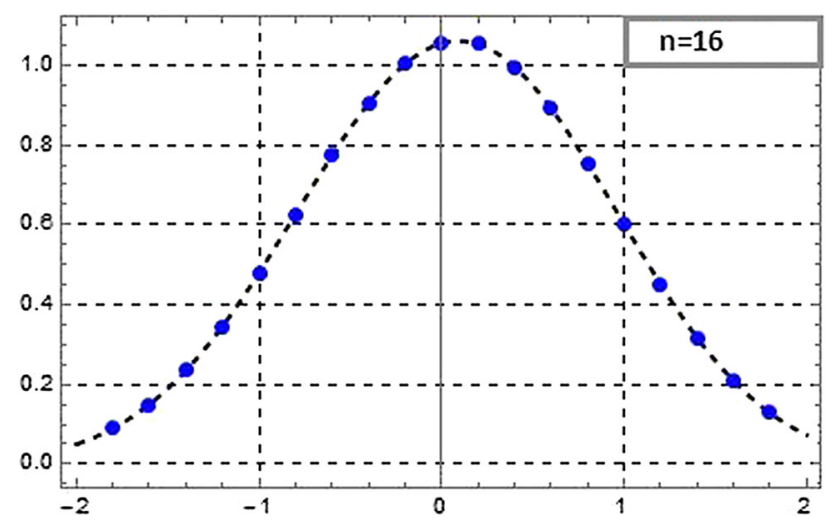

Fig. 2 Approximate solution for Example 1 using Chebyshev base with $n=16$

Example 2 Consider the following nonlinear integral equation:

$u(t)=f(t)+\int_{0}^{1} k(t, s)(u(s))^{-1} \mathrm{~d} s$,

where

Table 1 Numerical results of Example 2 using proposed method

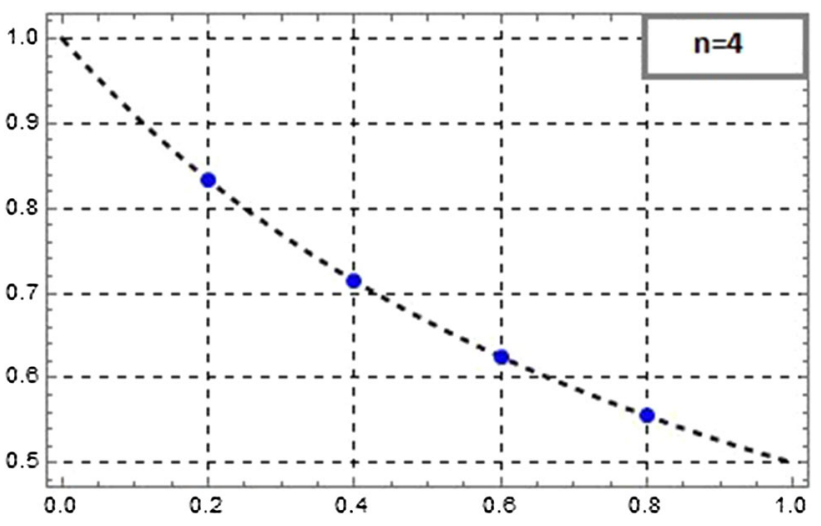

Fig. 3 The Chebyshev approximation for Example 2 using Chebyshev base with $n=4$

$$
\begin{gathered}
f(t)=\frac{21-11 \exp (10)}{100} \exp (-10(1+t))+\frac{1}{1+t}, \\
k(t, s)=\exp (-10(t+s)),
\end{gathered}
$$

with the exact solution $u(t)=\frac{1}{1+t}$. The obtained results of Example 2 with respect to proposed method in $[9,16]$ show that the mentioned method is powerful and give good numerical results in comparison with numerical scheme in $[9,16]$. As we expected, the presented method has produced highly numerical results. The best reported of error estimates for Bernstein method in [16] is $O(-16)$ for $n=10$, whereas we can achieve nearly equivalent error with $n=4$. The error estimates of the presented method for $n=4$, Bernestein method and Sinc method for $n=10$ have been represented in Table 1. Obtained numerical results for Example 2 can be seen in Fig. 3.

Example 3 Consider the following nonlinear integral equation:

\begin{tabular}{llll}
\hline$t$ & \multicolumn{2}{l}{ Absolute error } & \\
\cline { 2 - 4 } & Presented method $(n=4)$ & Bernestein Method $(\mathrm{n}=10)$ & Sinc Method $(\mathrm{n}=10)$ \\
\hline 0 & 0 & 0 & $7.77 \times 10^{-3}$ \\
0.1 & $4.44 \times 10^{-16}$ & $2.22 \times 10^{-16}$ & $2.85 \times 10^{-3}$ \\
0.2 & 0 & $3.33 \times 10^{-16}$ & $1.04 \times 10^{-3}$ \\
0.3 & 0 & $3.33 \times 10^{-16}$ & $3.86 \times 10^{-4}$ \\
0.4 & 0 & $1.11 \times 10^{-16}$ & $1.42 \times 10^{-4}$ \\
0.5 & 0 & 0 & $5.22 \times 10^{-5}$ \\
0.6 & 0 & $1.11 \times 10^{-16}$ & $1.92 \times 10^{-5}$ \\
0.7 & 0 & $1.11 \times 10^{-16}$ & $7.07 \times 10^{-6}$ \\
0.8 & $1.11 \times 10^{-16}$ & $1.11 \times 10^{-16}$ & $2.60 \times 10^{-6}$ \\
0.9 & 0 & 0 & $9.56 \times 10^{-7}$ \\
1 & 0 & 0 & $3.52 \times 10^{-7}$ \\
\hline
\end{tabular}




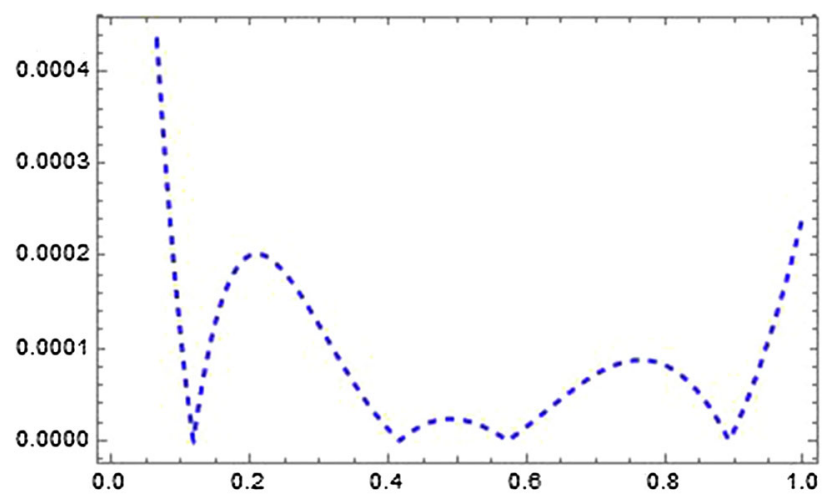

Fig. 4 The approximation absolute errors of Example 3 using Chebyshev base with $n=4$

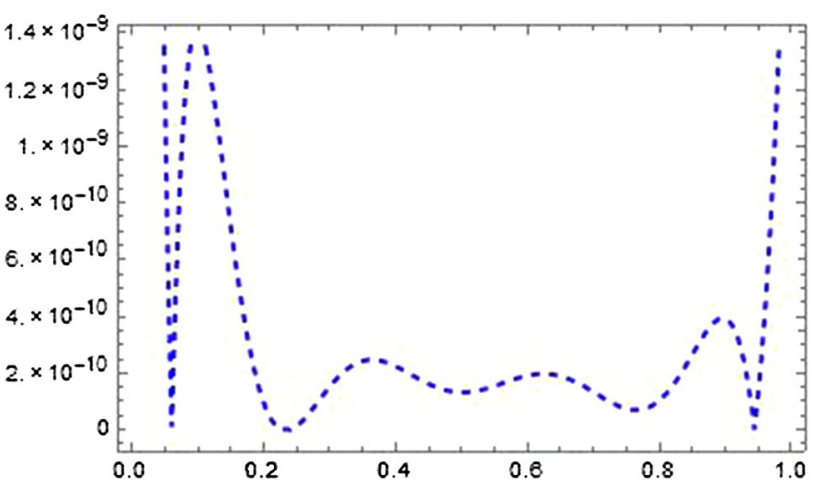

Fig. 5 The approximation absolute errors of Example 3 using Chebyshev base with $n=8$

$u(t)=f(t)+\int_{0}^{1} k(t, s)(u(s))^{-1} \mathrm{~d} s$,

where

$f(t)=\exp (-t)+2(-3+\exp (1)) \cos (t), \quad k(t, s)=\cos (t) s^{3}$,

with the exact solution $u(t)=\exp (-x)$. This problem has been solved by proposed scheme, as we expected good numerical results are obtained and the error behaviors of the presented method with $n=4,8$ have been shown in Figs. 4 and 5. As we can see, the reported absolute errors for $n=4,8$ are $O(-4)$ and $O(-10)$, respectively.

\section{Conclusion}

Being able to efficiently solve the problems in modeling chemical phenomenon that can be formulated as nonlinear integral equations is important. In this research, an efficient and accurate numerical scheme was presented for solving nonlinear integral equations and COSMO-RS integral equation which appeared in chemical phenomenon. By using the Chebyshev polynomials, the main problem was converted to a problem of solving a nonlinear system of algebraic equation. Comparing our numerical results and other numerical experiments, we may conclude the high accuracy of the presented method. With the availability of this methodology, it might be used to solve the other types of applied integral equations in other sciences.

Open Access This article is distributed under the terms of the Creative Commons Attribution 4.0 International License (http://creative commons.org/licenses/by/4.0/), which permits unrestricted use, distribution, and reproduction in any medium, provided you give appropriate credit to the original author(s) and the source, provide a link to the Creative Commons license, and indicate if changes were made.

\section{References}

1. Agarwal, R.P., Regan, D.O.: Ordinary and Partial Differential Equations. Springer, Berlin (2009)

2. Atkinson, K.E.: The numerical solution of a nonlinear boundary integral equation on smooth surface. IMA J. Numer. Anal. 14, 461-483 (1994)

3. Attary, M.: An extension of the Shannon Wavelets for numerical Solution of Integro-Differential Equations. In: Advances in Real and Complex Analysis with Applications, pp.277-288. Springer (2017)

4. Chapra, S.C., Canale, R.P.: Numerical Methods for Engineers. McGraw-Hill, New York (2010)

5. Fariborzi Araghi, M.A., Kazemi Gelian, G.: Numerical solution of nonlinear Hammerstein integral equations via Sinc collocation method based on double exponential transformation. Math. Sci. 7, 30 (2013)

6. Ghoreishi, F., Hadizadeh, M.: Numerical computation of the Tau approximation for the Volterra-Hammerstein integral equations. Numer. Algorithm 52, 541-559 (2009)

7. Hashemizadeh, E., Rostami, M.: Numerical solution of Hammerstein integral equations of mixed type using the Sinc-collocation method. J Comput. Appl. Math. 279, 31-39 (2015)

8. Madbouly, N.M., McGhee, D.F.: Adomian's method for Hammerestein integral equations arising from chemical reactor theory. Appl. Math. Comput. 117, 241-249 (2001)

9. Maleknejad, K., Alizadeh, M.: An efficient numerical scheme for solving Hammerestein integral equation arisen in chemical phenomenon. Procedia Comput. Sci. 3, 361-364 (2011)

10. Maleknejad, K., Hashemizadeh, E.: Numerical solution of the dynamic model of a chemical reactor by Hybrid functions. Procedia Comput. Sci. 3, 8-12 (2011)

11. Maleknejad, K., Hashemizadeh, E.: A numerical approach for Hammerstein integral equations of mixed type using operational matrices of hybrid functions. Politehn. Univ. Bucharest Sci. Bull. Ser. A Appl. Math. Phys. 73, 95-104 (2011)

12. Maleknejad, K., Hashemizadeh, E., Basirat, B.: Computational method based on Bernstein operational matrices for nonlinear Volterra-Fredholm-Hammerstein integral equations. Commun. Nonlinear Sci. Numer. Simul. 17, 52-61 (2012)

13. Maleknejad, K., Nedaiasl, K.: A sinc quadrature method for the Urysohn integral equation. J. Integral Equ. Appl. 3, 407-429 (2013)

14. Maleknejad, K., Nedaiasl, K.: Application of Sinc-collocation method for solving a class of nonlinear Fredholm integral equations. Comput. Math. Appl. 62, 3292-3303 (2011) 
15. Sahlan, M.N., Feyzollahzadeh, H.: Operational matrices of Chebyshev polynomials for solving singular Volterra integral equations. Math. Sci. 11, 165171 (2017)

16. Sahu, P.K., Saha, S.: Ray, Comparative experiment on the numerical solutions of Hammerestein integral equation arising from chemical phenomenon. J. Comput. Appl. Math. 291, 402-409 (2016)
17. Sweere, A.J.M.: Pair Configurations to Molecular Activity Coefficients. PAC-MAC (2017)

Publisher's Note Springer Nature remains neutral with regard to jurisdictional claims in published maps and institutional affiliations. 